\title{
VII. MICROBIOLOGIA DO SOLO
}

\section{HERBICIDAS NA NODULAÇÃO E NA ATIVIDADE DA NITROGENASE EM CULTURA DE AMENDOIM (')}

\author{
MARIA DO CARMO S. SOARES NOVO (2), LUCIANO SOUZA PAES CRUZ (3), \\ EDILBERTO PRINCE PORTUGAL (4), VIOLETA NAGAI (5) \\ e MANOEL CARLOS AZEVEDO ORTOLAN (6)
}

\section{RESUMO}

Com o objetivo de estudar os efeitos de trifluralin, metolachlor $\mathrm{e}$ linuron, aplicados, respectivamente, em pré-plantio-incorporado, pré-emergência e em pós-emergência, sobre o peso de matéria seca e número de nódulos e sobre a atividade da nitrogenase, foi conduzido um experimento de campo, em cultura de amendoim (Arachis hypogaea cv. Tatu) plantada em rotação com cana-de-açúcar. Os resultados das avaliaçōes feitas aos 30 , 50 e 80 dias após a germinaçăo mostraram nảo haver diferença entre tratamentos inoculados e não inoculados. Linuron foi o único herbicida prejudicial à nodulação, tendo afetado o número e o peso da matéria seca de nódulos, nas diferentes amostragens, e inibido a fixação do nitrogênio (estimada pela técnica da reduçáo do acetileno). Não foi observado efeito prejudicial dos herbicidas metolachlor e trifluralin, na nodulaçăo e na atividade da nitrogenase.

Termos de indexaçáo: amendoim, atividade da nitrogenase, nodulaçăo, herbicidas.

\section{ABSTRACT \\ INFLUENCE OF HERBICIDES IN NODULATION AND NITROGENASE ACTIVITIES IN PEANUTS CROP}

A field investigation was carried out to determine the influence of herbicides on peanuts (Arachis hypogaea cv. Tatu) Bradyrhizobium sp. symbiosis. The herbicides studied were trifluralin, metolachlor and linuron,

(1) Trabaiho financiado parcialmente pela FAPESP. Recebido para publicaçăo em 3 de outubro de 1989 e aceito em 6 de abril de 1990.

(2) Seçăo de Microbiologia do Solo, Instituto Agronómico (IAC), Caixa Postal 28, 13001 Campinas (SP).

(3) Seçáo de Fisiologia (IAC).

(4) Universidade Estadual de Campinas, Caixa Postal 6171, 13082, Campinas (SP).

(5) Seçáo de Técnica Experimental e Cálculo (IAC).

(6) Cooperativa dos Plantadores de Cana do Oeste do Estado de Sáo Paulo, Caixa Postal 142, 14160 Sertâozinho (SP). 
applied, respectively, in pre-planted-incorporated, pre-emergence and post-emergence. The results evaluated 30,50 and 80 days after germination, showed no difference between inoculated and non inoculated treatment. Linuron was the only herbicide that caused damage to nodulation, affecting the number and dry weight nodules and also inhibiting the nitrogen fixation (determined by nitrogenase activity). Metolachlor and trifluralin showed no damage effecis on nodulation and in the nitrogenase activity.

Index terms: peanuts, nitrogenase activity, nodulation, herbicides.

\section{INTRODUÇÃO}

O elevado preço da terra e os aumentos constantes dos custos de produção estāo exigindo a elevação dos rendimentos agrícolas e um aproveitamento mais racional do solo. Na regiāo de Ribeirão Preto, está sendo utilizado o sistema de rotação de cultura para a cana-de-açúcar, com a finalidade de ocupar a área de reforma do canavial que ficaria improdutiva por muitos meses.

$O$ amendoim tem sido usado em rotação com cana, pois adapta-se às necessidades dos produtores locais que desejam uma cultura de ciclo rápido, rentável, e que possa ser colhida na época da reinstalação do canávial. Levantamento da nodulaçāo do amendoim, nessas áreas, mostrou que, embora as sementes usadas no plantio não fossem inoculadas, havia uma populaçāo infectiva no local.

No entanto, a infestação da área de plantio por plantas daninhas é uma das causas da baixa produção dessa cultura: o período crítico de competição entre as plantas e 0 amendoim abrange o tempo em que o terreno não está coberto pela planta. Essa população deve então ser controlada, ou por cultivo mecânico ou por herbicida.

Um dos aspectos não muito conhecidos nessa cultura são os efeitos de herbicidas sobre a nodulação. Muito se questiona sobre os possiveis danos que esses produtos possam causar à bactéria do gênero Rhizobium e a outros microrganismos do solo.

São poucos os trabalhos em nossas condições sobre esse assunto e, para a cultura do amendoim, nada se conhece. Procurou-se, então, verificar o efeito dos herbicidas trifluralin (que é o mais empregado para essa cultura), metolachlor e linuron aplicados, respectivamente, em pré-plantio-incorporado, pré-emergência e pós-emergência, sobre a atividade da nitrogenase e sobre a população de Bradyrhizobium sp. existente e introduzida no local.

\section{MATERIAL E MÉTODOS}

O experimento foi conduzido em condiçōes de campo, na Fazenda Panorama, no município de Sertãozinho, SP, em latossolo roxo de textura argi- 
losa com as seguintes caracteristicas químicas: $P 17 \mu \mathrm{g} / \mathrm{cm}^{3}$, matéria orgânica $3,1 \%, \mathrm{pH} \mathrm{CaCl}_{2} 5,2, \mathrm{~K}+0,18 \mathrm{meq} / 100 \mathrm{~cm}^{3}$ de terra fina seca ao ar (TFSA), $\mathrm{Ca}^{2+} 2,6 \mathrm{meq} / 100 \mathrm{~cm}^{3}$ TFSA, $\mathrm{Mg}^{2+}{ }^{2} 0,9 \mathrm{meq} / 100 \mathrm{~cm}^{3}$ TFSA, $\mathrm{H}+$ $+\mathrm{Al}^{3}+3,4 \mathrm{meq} / 100 \mathrm{~cm}^{3}$ TFSA, S $3,7 \mathrm{meq} / 100 \mathrm{~cm}^{3}$ TFSA, T $7,1 \mathrm{meq} / 100 \mathrm{~cm}^{3}$ TFSA e $V 52 \%$. O solo foi preparado de modo convencional com araçāo e gradagens; para sua correção, realizaram-se calagem e adubação. O plantio do amendoim foi efetuado em 5/12/86, estudando-se o cultivar Tatu.

O delineamento experimental foi em blocos ao acaso, com parcelas subsubdivididas, com cinco repetiçōes. Nas parcelas estudaram-se os tratamentos com e sem inoculaçăo, preparando-se o inoculante com a mistura destas estirpes de Bradyrhizobium sp.: SMS-319, SMS-400 e SMS-561. Nas subparcelas, foram estudados os herbicidas trifluralin $(1,068 \mathrm{~kg} / \mathrm{ha})$ aplicado em pré-plantio-incorporado, metolachlor $(2,52 \mathrm{~kg} / \mathrm{ha})$, em pré-emergência, linuron $(1,20 \mathrm{~kg} / \mathrm{ha})$, em pós-emergência, e mais um tratamento, testemunha, sem herbicida; nas subsubparcelas, estudaram-se as épocas de amostragem.

Como parcelas experimentais, utilizaram-se canteiros com quatro linhas de amendoim, espaçados de 0,60m e com $20 \mathrm{~m}$ de comprimento.

Aplicaram-se os herbicidas com pulverizador costal de precisão, dotado de barra com dois bicos de jato plano da série 80.03 , com consumo de calda correspondente a $280 \mathrm{~V} / \mathrm{ha}$. $\mathrm{O}$ trifluralin foi incorporado uma hora após a aplicação, com grade de arraste; a aplicação do metolachlor foi imediatamente ao plantio e, a do linuron, após trinta dias.

Avaliou-se o efeito dos tratamentos no desenvolvimento da planta e na fixação biológica do nitrogênio aos 30,50 e 80 dias do plantio, sendo a primeira avaliação (aos 30 dias) realizada três horas depois de aplicado o linuron. Em cada época de amostragem, avaliaram-se o peso da matéria seca de nódulos, o número de nódulos e a atividade da nitrogenase pela técnica da redução do acetileno (HARDY et al., 1968). Para a análise da redução do acetileno, usaram-se três raizes por parcela e, após o período de incubação, retiraram-se três amostras de cada parcela.

Para a análise da variância, os dados de número de nódulos foram transformados em $\sqrt{x+0,5}$; os testes de comparação de médias foram feitos com os dados transformados; no entanto, nos quadros, as médias são apresentadas na escala original. Empregou-se o teste de Tukey para comparação múltipla entre os herbicidas e, o de Dunnett, para comparar os herbicidas com a testemunha. A significância do contraste entre tratamentos com e sem inoculaçāo foi verificada com o teste $F$.

\section{RESULTADOS E DISCUSSÃO}

Nenhum dos herbicidas utilizados mostrou efeito fitotóxico nas plantas de amendoim, não sendo observadas anomalias ou modificaçōes no aspecto morfológico. 
Os tratamentos não inoculados estavam bem nodulados e em todos os tratamentos a maioria dos nódulos foi de tamanho pequeno. DIATLOFF \& LANGFORD (1975) afirmam que é difícil superar a nodulação da população natural com estirpes introduzidas, mesmo em solo com baixo número de rizóbios, o que também foi observado neste trabalho, como se pode ver nos quadros 1 e 2.

Aos 50 dias, pode-se observar que houve efeito negativo da inoculação quanto ao número de nódulos, onde se aplicou linuron, tendo havido uma redução de $42,9 \%$ para esse parâmetro em relaçāo ao tratamento com linuron e com a população nativa, mostrando assim maior tolerância dessa população ao herbicida.

ALAA-ELDIN et al. (1981) relataram diminuição no número de nódulos, em ensaio de vaso, em soja tratada com esse produto,e BOLLICH et al. (1985) mostraram que, em ensaio de campo, na cultura de soja, linuron a 1,76kg/ha provocou efeito ocasional na nodulação.

Comparando-se os herbicidas entre si e em relaçāo à testemunha Quadro 1 - verifica-se que, aos 30 dias e nos tratamentos nāo inoculados aos 50 dias, não houve diferença entre herbicidas, nem estes diferiram da testemunha; aos 50 dias, porém, no tratamento inoculado, o número de nódulos observado no linuron e trifluralin foi estatisticamente inferior ao do metolachlor. LOPES et al. (1971) relataram a ausência de prejuízo pelo trifluralin na nodulação do feijoeiro, avaliado aos 55 dias, e GIARDINI et al. (1979) observaram que, em avaliaçōes aos 35,58 e 98 dias em soja, trifluralin e alachlor, que tem modo de ação semeIhante à do metolachlor, nāo prejudicaram a nodulação.

Aos 80 dias, em comparação com a testemunha, verifica-se que os herbicidas contribuiram para que houvesse redução no número de nódulos nas plantas de amendoim, porém não houve diferença entre os tratamentos com herbicidas.

No quadro 2, encontram-se as médias dos pesos de matéria seca de nódulos formados nas raizes de amendoim. Verifica-se que, em relação à testemunha, houve sensivel redução no peso de nódulos nos tratamentos com linuron aos 50 e 80 dias e que, aos 50 dias, linuron reduziu o peso de nódulos, diferindo significativamente do trifluralin e do metolachlor. Aos 80 dias, o peso da matéria seca de nódulos da testemunha nāo diferiu do observado no tratamento com trifluralin e foi superior ao do linuron e do metolachlor. Os resultados concordam com DUNIGAN et al. (1972), segundo os quais linuron a 1,12kg/ha reduziu a massa de nódulos de plantas de soja, em dois tipos de solo. NEVES (1983), em ensaio de campo com feijāo e caupi, observou que, como os nódulos são formados nas primeiras semanas após a germinação, qualquer efeito tóxico dos herbicidas sobre as bactérias ou sobre a planta hospedeira, nesse periodo, pode prejudicar irreversivelmente o estabelecimento de uma nodulação eficiente, diminuindo a disponibilidade de $\mathrm{N}_{2}$ fixado para as plantas. 


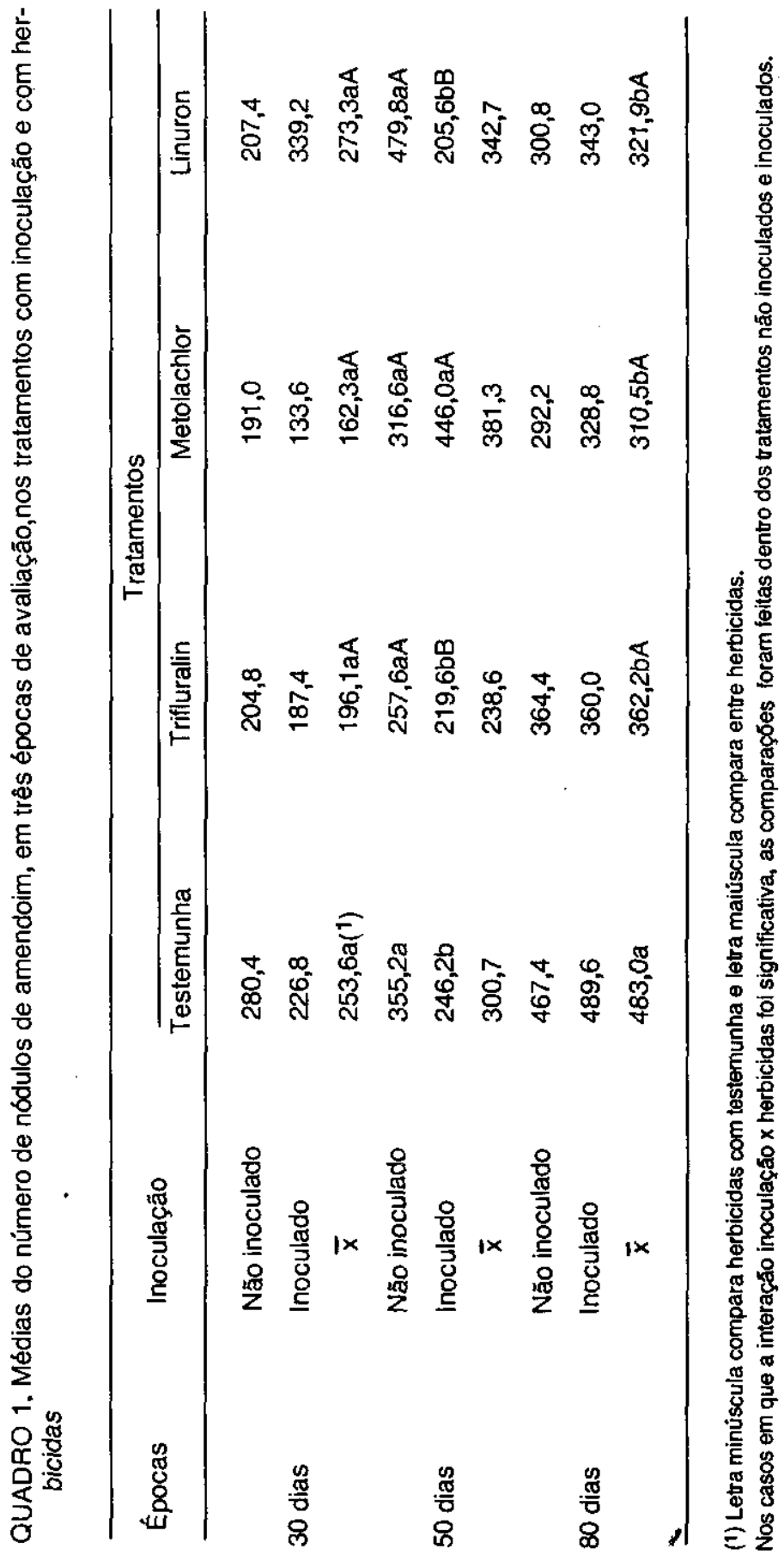




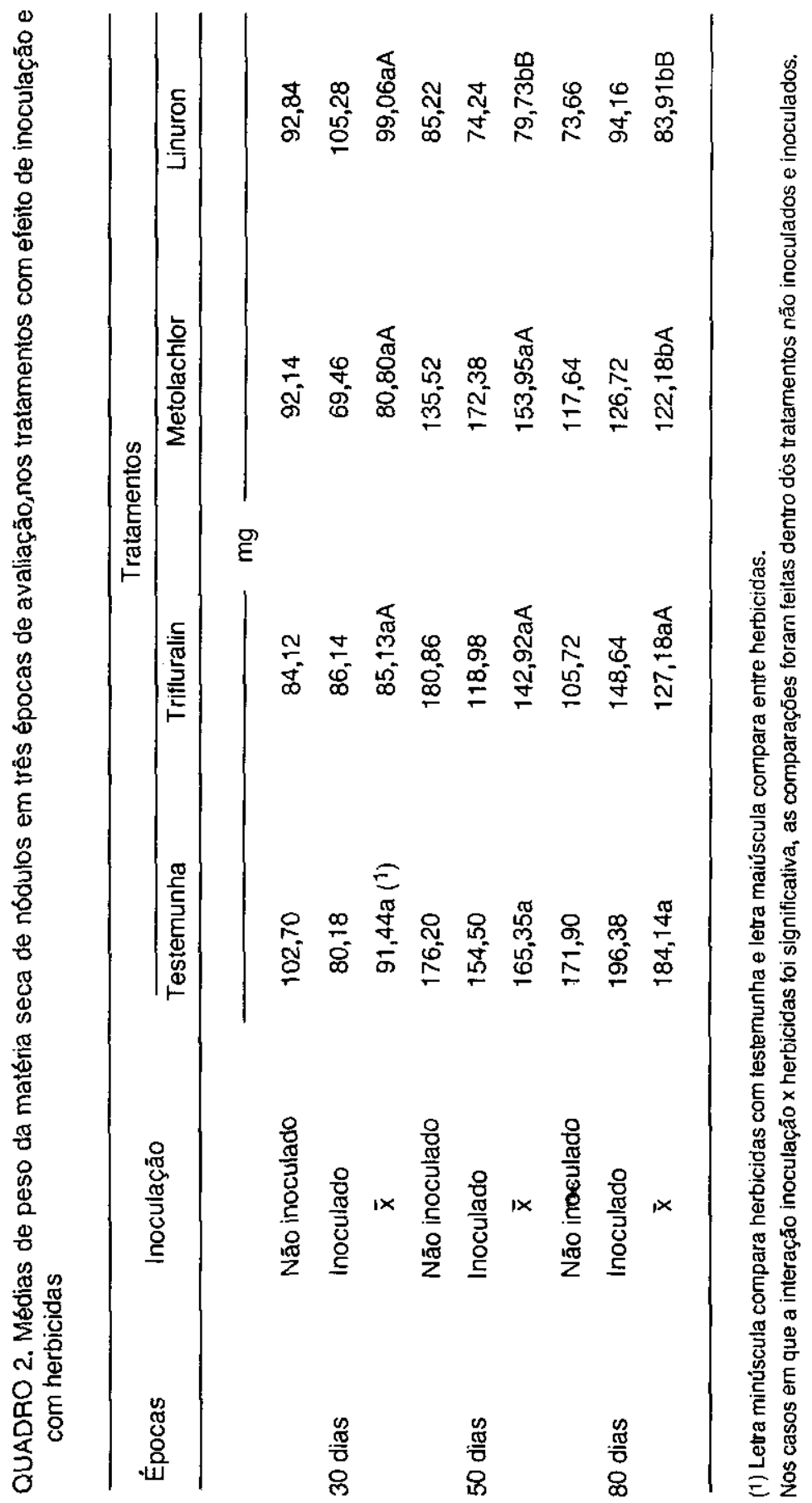




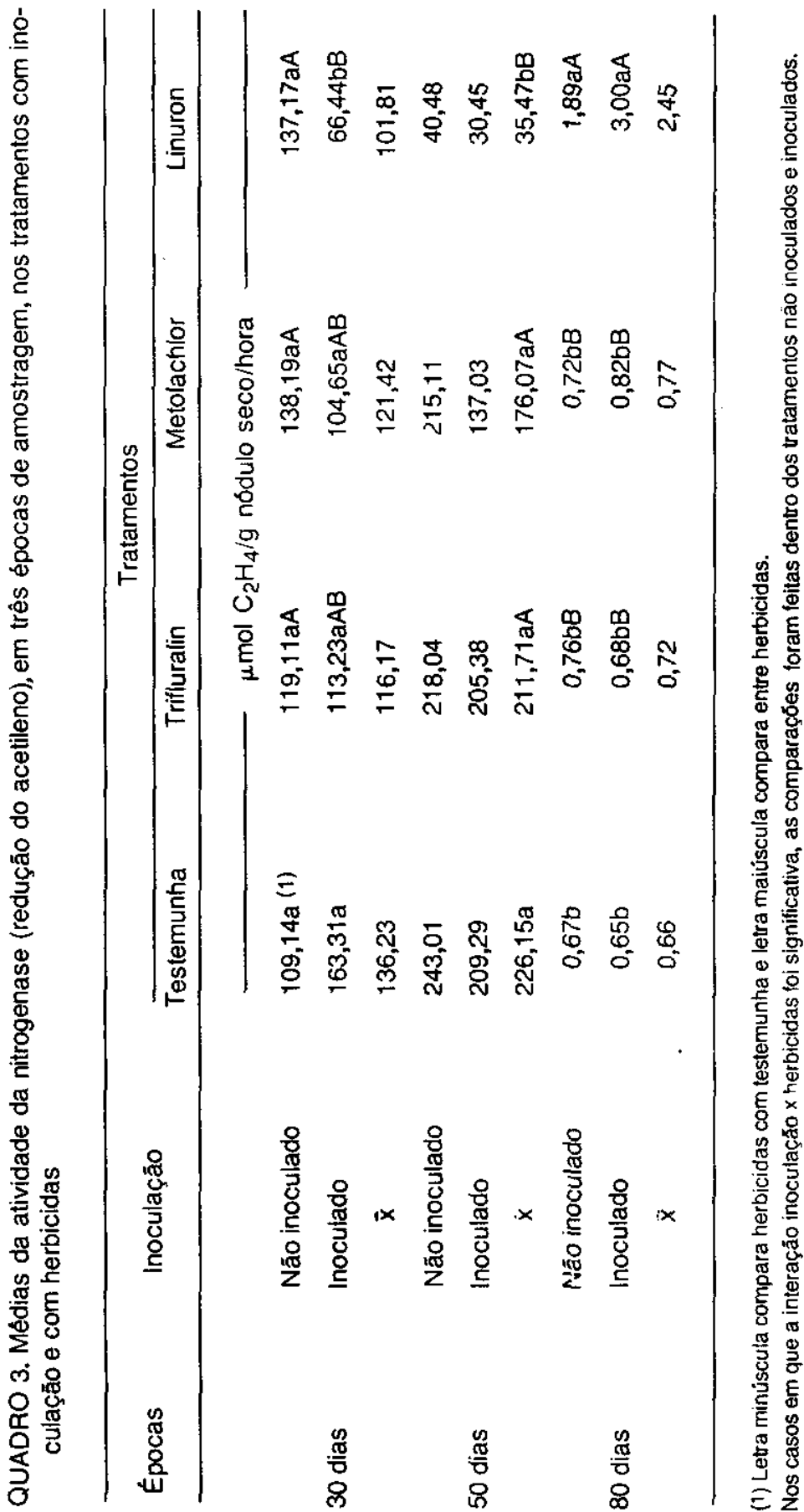


Observa-se, no quadro 3 , que nos resultados dos tratamentos na reduçāo do acetileno a etileno em plantas de amendoim, à exceção do tratamento com linuron, houve aumento na atividade da nitrogenase até os 50 dias, sendo, aos 80 dias, a fixação muito baixa. Aos 30 dias no tratamento inoculado; tinuron causou diminuição na fixação do nitrogênio, ocorrendo o inverso aos 80 dias. Aos 50 dias, o tratamento inoculado resultou em diminuição na atividade da nitrogenase. De modo geral, a população autóctone foi mais eficiente na fixação simbiótica do nitrogênio.

Observa-se, também, que, aos 30 dias, no tratamento sem inoculação, não houve diferença entre herbicidas e estes não diferiram também da testemunha. Na mesma amostragem, nos tratamentos inoculados, em relaçāo à testemunha; linuron levou à menor atividade da nitrogenase $e$, conseqüentemente, à baixa fixação do nitrogênio, o que também ocorreu aos 50 dias. Aos 80 dias, embora a demanda de nitrogênio seja menor, pois os genóforos já estão formados,e a fixação seja pequena, tanto nos tratamentos inoculados como nos não inoculados, as plantas que receberam linuron em pós-emergência apresentavam maior fixação em relação aos outros tratamentos. No inoculado, essa fixação foi 4,6 vezes maior que a fixação da testemunha. RENNIE \& DUBETZ (1984) verificaram que linuron aplicado em pré-emergência, na cultura da soja, reduziu significativamente a fixação do nitrogênio, enquanto trifluralin estimulou a fixação no primeiro aro e não teve nenhum efeito no ano seguinte. BOLLICH et al. (1985) relataram que a aplicação de alachlor $(2,2 \mathrm{~kg} / \mathrm{ha})$ em solo arenoso acarreta diminuição na produção de $\mathrm{C}_{2} \mathrm{H}_{4}$ /planta/hora, $\mathrm{O}$ que não ocorreu nesse caso até 50 dias. $\mathrm{O}$ motivo pelo qual o linuron afetou diretamente a fixação do nitrogênio pode ser devido a seu modo de ação. De acordo com HOLST et al. (1982) e MUSCHINEK et al. (1979), linuron afeta as reações fotossintéticas, inibindo a reação de Hill e a fixação de dióxido de carbono, afetando diretamente a fixação de $\mathrm{N}_{2}$.

\section{CONCLUSOẼS}

1. Não houve efeito de inoculação;

2. Houve redução na matéria seca de nódulos e na atividade da nitrogenase; o número de nódulos não foi afetado significativamente;

3. O linuron inibiu significativamente a fixaçāo do nitrogênio.

4. A nodulação e a atividade da nitrogenase não foram, de modo geral, afetados por metolachlor e trifluralin.

\section{REFERÊNCIAS BIBLIOGRÁFICAS}

ALAA-ELDIN, M.N.; MAHMOUD, S.A.Z.; MAKAWI, A.; ABDEL-NASSER, M. \& HERZALLAH, N.A. Effect of pre-emergence application of some herbicides on nodulation, nitrogen fixation and growth of soybean. Pesquisa Agropecuaria Brasileira, Brasilia, 16(6):833-839, 1981. 
BOLLICH, P.K.; DUNIGAN, E.P. \& JAI, W.N. Effects of seven herbicides on $\mathrm{N}_{2}\left(\mathrm{C}_{2} \mathrm{H}_{2}\right)$ fixation by soybeans. Weed Science, Champaign, 33(4):427-430, 1985.

DIATLOFF, A. \& LANGFORD, S. Effective natural nodulation of peanuts in Queensland. Queensland Joumal of Agricultural and Animal Sciences. Brisbane, 32(1):95-100, 1975.

DUNIGAN, E.P.; FREY, J.P.; ALLEN JUNIOR, L.D. \& MCMAHON, A. Herbicidal effects on the nodulation of Glycine max (L.) Merril. Agronomy Joumal, Madison, 64(6):806-808, 1972.

GIARDINI, A.R.; LOPES, E.S. \& DEUBER, R. Influência de herbicidas na nodulação da soja (Glycine max (L.) Merril). Planta Daninha, Piracicaba, 2(1):21-33, 1979.

HARDY, R.W.F.; HOLSTEN, R.D.; JACKSON, E.K. \& BURNS, R.C. The acetylene-ethylene asssay for $\mathrm{N}_{2}$ fixation: laboratory and field evaluation. Plant Physiology, Lancaster, 43(8):1185-1207, 1968.

HOLST, R.W.; YOPP, J.H. \& KAPUSTA, G. Effect of several pesticides on the growth and nitrogen assimilation of the Azolla-Anabaena symbiosis. Weed Science, Champaign, 30(1):54-58, 1982.

LOPES, E.S.; DEUBER, R.; FORSTER, R.; GARGANTINI, H. \& BULISANI, E.A. Influência dos herbicidas EPTC e trifluralin e da inoculação das sementes com Rhizobium phaseoli na nodulação e produçăo do feijoeiro (Phaseolus vulgaris L.) Bragantia, Campinas, 30(11):109-116, 1971.

MUSCHINEK, G.; GARAB, G.I.; MUSTÁRDY, L.A. \& FALUDI-DÁNIEL, A. The mechanism of linuron phytotoxicity in mayze. Weed Research, Oxford, 19(2):101-107, 1979.

NEVES, M.C.P. Efeitos de herbicidas na nodulaçăo, fixaçăo de nitrogênio e produção de feijāo (Phaseolus vulgaris) e caupi (Vigna unguiculata). Arquivos da Universidade Federal Rural do Rio de Janeiro, Itagual, 6(2):131-139, 1983.

RENNIE, R.J. \& DUBETZ, S. Effect of fungicides and herbicides on nodulation and $\mathrm{N}_{2}$ fixation in soybean fields lacking indigenous Rhizobium japonicum. Agronomy Journal, Madison, 76(3):451-453, 1984. 